\title{
When is a Drummer not a Drummer? Developing Coordination, Musicianship, and Creativity through Electronic Drum Performance
}

\author{
Bryden Stillie
}

\section{Introduction}

If asked to describe the instrument a drummer plays, most people would probably describe an acoustic drum kit, consisting of a bass drum, snare drum, tom toms, and cymbals. It is unlikely that the words "hybrid" or "electronic" would be part of the language used. The technologies related to drumming have seen significant advancement since the first electronic drum kits in the early 1980s, and these technologies have played a significant role in shaping the way music sounds today. Throughout this chapter I refer to three different types of drum kit setup; these are as follows: (1) "acoustic," the traditional drum kit setup; (2) "electronic," pads/triggers used to activate electronic sounds from a sound module or computer; and (3) "hybrid," a setup containing a mixture of acoustic drums, electronic pads/triggers, and/or sample pads $^{1}$ or percussion controllers (Figure 13.1). ${ }^{2}$

At the age of 14 I received two Christmas gifts that would go on to define my career in musical performance and drum kit teaching. The first was the drum kit instructional VHS tape, Bruford and the Beat (Bruford 1982). The second was a Yamaha DD-11 digital drum kit. The opening scene of the instructional video shows drummer Bill Bruford playing his hybrid drum kit, which combined a Tama acoustic drum kit and Simmons ${ }^{3}$ electronic drum kit. The sound produced from the first three pads he played (a total of fourteen notes) became the inspiration for my approach to teaching creative use of drum kit related music technology, in my current role as Senior Lecturer in Music on the BA Popular Music program at Edinburgh Napier University, Scotland.

This chapter presents and examines an innovative approach to teaching drum kit related technologies through the creation of a solo electronic drum performance undertaken by my Year 3 (undergraduate) drum kit students. The project requires students to recreate a song of their choosing, using music creation software, ${ }^{4}$ and then perform 


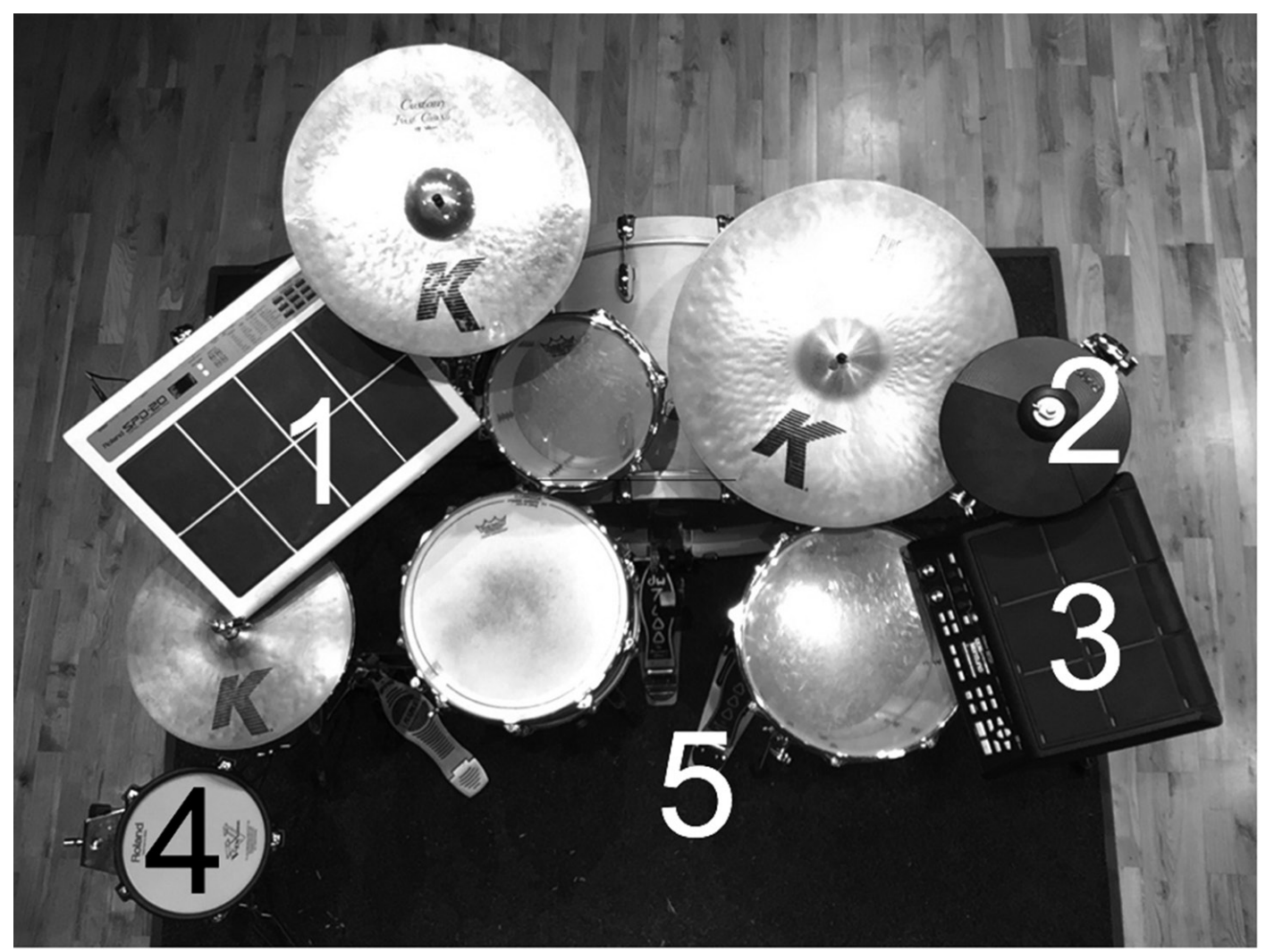

Figure 13.1 A hybrid drum kit setup containing: (1) Roland SPD-20 percussion controller, (2) Roland CY-5 cymbal trigger pad, (3) Roland SPD-SX sample pad, (4) Roland PD-85 mesh head trigger pad, and (5) Roland KD-7 kick drum trigger.

the song using a Roland SPD- $20^{5}$ percussion controller, music software, and external trigger pads. ${ }^{6}$ My motivations for designing the project outlined in this chapter were that I wanted to: equip my students with skills and knowledge that enabled them confidently to integrate and implement drum kit technologies in live performance settings; increase students' versatility (Smith 2014: 38) and subsequent employability; and encourage them to become more creative as musicians. I developed a course that equips my drum kit students with highly relevant skills and knowledge required by the modern drummer.

\section{The study of drum kit in higher popular music education}

Drum kit is taught as a principal study within most undergraduate programs that identify as teaching popular music performance. Drum kit is also taught on programs that focus specifically on jazz and, in some cases, the instrument is also taught to percussion students studying on traditional Western art music programs. The learning experiences of drummers on these different program types may be markedly different due to the different approaches used to teach the instrument, ${ }^{7}$ and the expectations placed on 
drummers at different stages of degree programs. These differences have a significant influence on the design of drum kit curricula, causing disparity in both depth and breadth of learning achieved by drummers graduating from different institutions.

Drum kit instructors tend to assemble curricula from a series of highly established compartmentalized teaching approaches and methods (Dean 2012; Smith 2013) that are firmly rooted in developing technical skills through rudiments, coordination exercises, and application of rhythmic concepts, applied to the acoustic drum kit. Instructors decide on what to include in their curricula, based on factors including areas of student interest, course/program outcomes, personal values, student goals and skill sets, emerging trends, and the levels and abilities of learners. It is difficult to define a single curriculum that all undergraduate drummers should follow. There are very few resources or established learning and teaching approaches that specifically focus on how to learn and creatively use drum kit related technologies. Given that there has been significant development in these technologies and that such technologies are becoming commonplace in the hybrid kit setups used by many modern drummers, ${ }^{8}$ there is a need to develop new pedagogical models (Smith 2014: 44) and curricula that support the teaching of drum kit technology-based performance.

\section{Educating students in drum kit technologies}

During my own experience of studying drum kit, I had only one lesson that explored using drum kit technologies. In that lesson, I did not get to set anything up - all I had to do was play a simple rock beat and strike a pad on beat one of each new bar to activate a percussion loop. Whilst I learned an approach to triggering samples (a skill that many drummers require), the experience omitted covering the knowledge needed to replicate, rebuild, or creatively use this technology. Anecdotally from colleagues, and from my experience as an External Examiner ${ }^{9}$ and guest lecturer, I have witnessed similar teaching approaches that only explore the "plug and play" features of drum kit technologies. Although these allow some technologies to be implemented quickly in performance, it means that their full functionality and affordances are rarely explored.

As noted above, there is a dearth of educational resources related to learning how to use drum kit technologies creatively. Learners often have to rely on the information provided in owner's manuals and technical demonstrations available through product manufacturers' websites, to learn the basic functionality of their equipment. During my search for relevant teaching resources, only two instructional books appeared to explore electronic drumming in any depth. The Beginner's Guide to Electronic Drums (Terry 2011) clearly defines and explains key terminology and approaches to integrating technologies related to electronic drums into hybrid kit setups. However, the book stops short of exploring creative and extended uses of the technologies to play anything other than percussion sounds. Fujiyama: Combining Acoustic and Electronic Drums (Jimbo 2003) also explores electronic drumming from a hybrid perspective. One advantage of Jimbo's approach to hybrid performance is that the drummer has some control over the 
tempo of their own performance. This differs from the electronic drum performance projects undertaken by my students, as they are required to play along to a metronomic click track; my reasons for this are explored later in this chapter. Jimbo provides a comprehensive method of notating musical performance for a hybrid kit setup. However, the notation is excessive and probably quite intimidating for drummers who do not read treble or bass clef, especially when all the pitched content of the music is activated by playing the single pattern indicated in the drum kit notation staff.

Both of the aforementioned books explore electronic drums from a hybrid kit perspective but are still firmly rooted in learning to activate electronic sounds by playing patterns that are recognizable as drum grooves. ${ }^{10}$ For most acoustic drum kit players, there are certain "go-to" positions to play grooves, for example, one stick playing on the hi-hat, one stick on the snare, and bass drum with one foot. Acoustic kit players therefore will likely find the transition to the type of hybrid performances advocated by Terry (2011) and Jimbo (2003) relatively easy, as they would be familiar with the coordination required to play the patterns. Electronic trigger setups ${ }^{11}$ remove the need to adhere to conventional coordination patterns, as any sound can be triggered from any pad. My approach introduces new coordination possibilities that are constrained only by the technical limitations of the selected percussion controller, software functionality, and the physical and technical limitations of the performer.

\section{The drum kit learning and teaching experience at Edinburgh Napier}

A key priority in Edinburgh Napier University's Strategy 2020 (Edinburgh Napier University n.d.) is to move toward a program-focused approach to learning, teaching, and assessment (Hartley and Whitefield 2011; McDowell 2012). This cohesive approach to program design requires modules (courses) to complement one another so that students can utilize skills and knowledge developed in one module to help meet the requirements of another. This approach ensures that students have opportunities to develop necessary skills and knowledge to engage with new learning as they progress through the program.

The BA Popular Music program at Edinburgh Napier is a four-year honors degree. The typical learning experience of a student on the program encompasses three areas of study, as follows: performance (solo and ensemble-based), musicology, and music technology. In performance modules, students develop: a strong working knowledge of music theory (approximate to London College of Music Popular Music Theory Grade $\mathrm{VII}^{12}$ ); music analysis skills; and high-level instrumental performance abilities. Students also learn four music production software packages. At the end of Year 1 students have developed competence using Logic Pro X, and have all earned the AVID ${ }^{13}$ Certification in Pro Tools (101 level) and Sibelius (101 and 110 level). ${ }^{14}$ Ableton Live is taught in Year 3 which compliments the timing of their electronic drum kit project. 
My Year 1 and 2 drum kit curriculum focuses on developing technical skills and coordination related to the acoustic drum kit. Set works in the areas of Latin, jazz fusion, and progressive rock are used to improve musicality, coordination, and independence. These are supplemented with snare drum pieces that enable drummers to learn and contextualize all forty Percussive Arts Society rudiments (Percussive Arts Society 1984). This provides a solid foundation on which to begin exploring more complex concepts covered in Year 3 , such as displacement, metric modulation, and electronic drumming. In Year 4, the curriculum is student-led and therefore highly personalized, enabling students to study individual topic areas related to their own drumming interests and future career ambitions.

\section{My electronic drumming curriculum and the student learning experience}

The curriculum that supports development of my students electronic drumming performance projects is delivered in a blended format (Garrison and Kanuka 2004), utilizing a flipped classroom approach (Bergmann and Sams 2012). This pedagogical framework contains three areas of learning and teaching activity: preparation, faceto-face activities, and post-class consolidation. The face-to-face and online learning are deeply interlinked through a series of learning experiences that provide the "scaffolding" (Meskill 2013: 13) to enable students to interact and learn collaboratively online. The online content is hosted on the university's virtual learning environment (VLE), Moodle. In semester one, a six-week block of learning and teaching experiences supports the curriculum (see Table 13.1).

Table 13.1 The Semester One Curriculum

\begin{tabular}{ll}
\hline Week & Curriculum Areas \\
\hline 1 & $\begin{array}{l}\text { Introduction and Icebreaker: Lecture to explain the course aims and outcomes, } \\
\text { and how the online content and resources are to be used. This then feeds the first } \\
\text { online discussion. }\end{array}$ \\
& $\begin{array}{l}\text { Accessing and editing internal sounds on the Roland SPD-20 and constructing } \\
\text { patches. }^{15}\end{array}$ \\
3 & Connect external triggers, pads, and controllers to the SPD-20. \\
4 & $\begin{array}{l}\text { MIDI Out: exploring how MIDI information from the SPD-20 can be used to } \\
\text { control music software. }\end{array}$ \\
5 & $\begin{array}{l}\text { Exploring advanced music software functionality and signal routing, and the MIDI } \\
\text { In capabilities of the SPD-20. }\end{array}$ \\
& $\begin{array}{l}\text { Using the electronic drum notation I have developed, students learn and perform a } \\
\text { sample-based }{ }^{16} \text { version of "Spitfire" (The Prodigy 2004) on a hybrid kit setup. }\end{array}$ \\
\hline
\end{tabular}




\section{Preparatory activities}

Students prepare for face-to-face classes by watching my video demonstrations of hardware and software functionality provided in the VLE. Digital delivery of this procedural and technical information ${ }^{17}$ which remains fairly consistent year to year has multiple benefits:

- Students learn at their own individual pace.

- Learning occurs asynchronously and content can be revisited to ensure students' full understanding before attending class (rather than passively absorbing information in class).

- The resources provide ongoing support.

- Face-to-face time can be more productively spent on active learning and experimentation.

\section{Face-to-face classes}

Face-to-face interactions occur in two formats - group learning experiences and tutorfacilitated workshops. In these settings, learning is "a highly social activity that takes place in realistic contexts and activities" (Selwyn 2011: 88). These practical, situatedlearning activities allow students to collaborate and support each other to develop greater practical understanding of hard- and software functionality. ${ }^{18}$ This is achieved through engaging with a series of problem-based learning activities I designed based on my own observations and on student feedback that identified curricular areas that they found challenging. These activities require students to troubleshoot and solve soft- and hardware issues by applying, linking, and demonstrating specific learning gained from the online content. These activities exemplify learning in Vygotsky's "Zone of Proximal Development," where through social interaction, learners are able to develop deeper understanding of the topics than if working individually (Vygotsky 1978: 86).

\section{Online consolidation activities and support}

In semester one, online collaborative learning occurs in Wikis, general discussion forums related to FAQs and to solving technical challenges, and through forums that are focused on case study analysis, where students develop a "community of practice" (Lave and Wenger 1991: 42). Students collaborate to create Wikis that explain the functionality of a variety of drum trigger types, which provides a knowledge base for the rest of the class. This activity is particularly useful as it allows class time to be focused on the more challenging aspects of the course, and as drum pads and triggers are generally non- 
proprietary, the knowledge created is transferable to drum kit technologies produced by almost any manufacturer. This ensures students can make informed choices regarding the triggers and pads they might use beyond the classroom environment. Additionally, it helps to foster "rich communities of inquiry" (Garrison and Anderson 2003: 5) where students become cocreators of knowledge. I use case studies that explore how students in previous cohorts created their projects, as stimuli for discussion forums. These case studies include videos of previous student performances that provide an indication of the expected standard of projects and the different ways in which technologies have been used.

In semester two, students develop their electronic performance over a fifteen-week period. Learning is self-directed, and students make use of substantial online resources to support the development of their project. Ongoing face-to-face and online support is provided by student mentors from Year 4 of the program who have firsthand experience of creating and performing projects of this nature. These mentors help the current students to overcome challenges faced in the development of their individual projects, making for a highly personalized learning experience. Students are expected to perform their projects in a group workshop late in the semester, serving as an opportunity to receive formative feedback. The electronic performance of the song created during this project is assessed during the student's summative performance exam at the end of semester two.

\section{Selecting software and hardware}

The use of digital audio workstation Logic Pro $\mathrm{X}$ is encouraged for several reasons: the prior experience students have using this software in the earlier years of their studies; its flexibility when routing and manipulating MIDI signals; and its wide range of preset synthesized sounds, effects, and audio loops that allow students to work creatively and achieve results quickly. However, due to the functionality of modern music software, a detailed knowledge of how signals are transferred, received, and processed between hardware and software is no longer required to create music. This means that in order to make full use of the available hardware and software students must learn and develop a working knowledge of how MIDI is used to connect and control devices, in order to realize their projects.

I selected the Roland SPD-20 ${ }^{19}$ (Figure 13.2) as the percussion controller and trigger interface used for these projects. Although other manufacturers such as Yamaha and Alesis produce similar percussion controllers, some of which have more advanced MIDI features, the MIDI functionality of the SPD-20 is simple to understand and the parameters are easy to access and edit. Additionally, the SPD-20's MIDI parameters are common to the controllers produced by other manufacturers which ensures that my students are learning transferrable knowledge and skills that can be applied to 


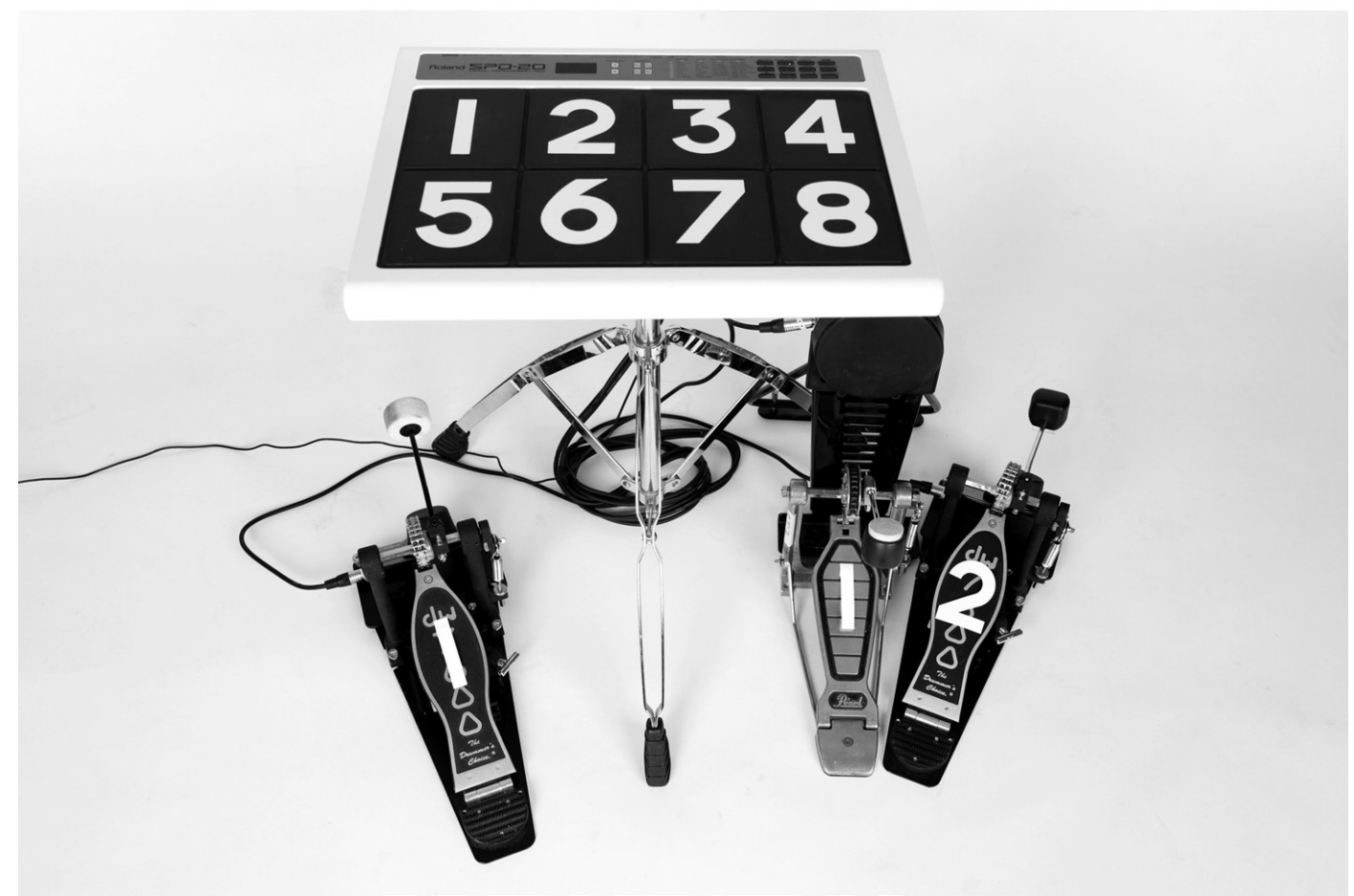

Figure 13.2 The SPD-20 with pad and trigger pedal numbering. Photograph by Alexandra Duncan.

different electronic drum kit equipment setups, and that they learn how these common MIDI parameters can be used to control music software in less orthodox ways ${ }^{20}$ as they develop their performance.

\section{How students develop their electronic performances}

Students are given freedom of choice to select a piece to perform; however, I approve their choice to ensure that it will pose an appropriate level of challenge both in the use of technology and to their coordination. Once their choices are agreed, there is a series of key stages to complete before students are able to perform their song using just the percussion controller and associated technologies.

Initially, each student has to create their own version of their chosen song in Logic Pro X (or their preferred music software package). They draw on aural skills to analyze the original recording to work out constituent parts of the arrangement, which they must then program using software instruments and/or record as audio. If original audio stems are available, students may be permitted to use a limited number of these to help construct the track, for example, original isolated vocal tracks. Students are encouraged to approach their projects creatively through remixing or rearranging the original 
material. During this process, students have to research, learn, and deploy genre-specific production techniques related to the musical style of their chosen arrangement.

Completed arrangements must next be deconstructed into playable parts. ${ }^{21}$ The physical layout of the trigger setup must now be considered together with how it will be used to activate the sonic elements required to successfully realize the performance. In designing the layout of the trigger setup, students must consider (1) how to activate the constituent parts of the arrangement using four limbs, (2) the technical and physical performance limitations imposed by software and hardware, and (3) the trigger equipment available. Sonic elements of a performance can be recreated using samples, live software instruments, or both. The sample-based approach could be used with almost any MIDI percussion controller, as only a single software sampler instrument needs to be activated. Percussion controllers, such as the SPD-20, that are capable of sending multiple MIDI notes on different MIDI channels from a single pad expand the performance possibilities to playing live instruments, activating automation, and rearranging the performance using more advanced software functionality.

When considering the approach to take, standard acoustic drum kit coordination no longer needs to dictate how the piece will be performed, as noted above. This does not mean that non-drummers could easily perform these pieces, however; the coordination required to play these arrangements is an extension of the coordination drummers already possess. The software and hardware afford any sound to be played with any limb, which opens up new possibilities and approaches to playing sounds that would normally be associated to a particular limb on a standard drum kit (e.g., the bass drum is ordinarily played with the right foot). A common approach is to play the drum pattern and bass line of the song with the feet, leaving the hands free to play the rest of the harmonic and melodic content using sticks.

The next stage is probably the most laborious - building multiple patches on the controller that contain the specific MIDI information required to activate desired sounds. Often, several patches are required to perform a single section of the song, sometimes two per bar, due to limits on the number of MIDI notes that can be sent from any patch and the need to access different sounds within bars. Additionally, the need (for playability) to achieve a somewhat consistent layout of sounds on the pads increases the number of patches required. For example, if there is a repeated musical phrase present in the song, it makes sense that the MIDI notes required to play the phrase appear in the same pad positions on different patches, making the performance easier to remember.

A key factor in realizing these projects is that the performance must be played to a metronomic click track, normally provided by the selected music software. This allows the music software to control patch changes at predetermined points using MIDI Program Changes. ${ }^{22}$ A MIDI track in the music software is populated with the Program Changes that reflect the way patches must be loaded to play the arrangement. This allows the performer to concentrate on playing through the song without having to physically change patches by pressing buttons. The Roland SPD-20 is able to react to Program Changes and set up new patches in a very short space of time, which makes 
it an excellent choice for this project. I have tested some other sample trigger pads and have found that they are unable to load samples quickly enough after receiving a Program Change message to allow sounds to be triggered on beat one of the next bar. Alternative approaches to changing patch ${ }^{23}$ exist, but they do not provide the timing accuracy required to realize these projects.

Following each of these stages, students prepare to perform the song. At this point an interesting phenomenon occurs; although the students are essentially learning a new piece of music, they have spent so long thinking about the way in which it will be performed that they have already learned most of the physical coordination patterns and, therefore, learn to perform the piece very quickly. The main challenge lies in fusing the sections of the song together so they can play the performance from start to finish. The final stage of the process is the performance of the song in the summative performance assessment. Having completed these projects students are proficient in the use of drum kit related technologies and many go on to feature the use of drum kit technology in their Year 4 exams the following year.

\section{Developing a notation standard and sharing projects}

In addition to the curriculum I designed, I have developed a musical notation method that allows my students to learn electronic performances I have created, and provides a way for students to share their own projects with others in the class. Figure 13.3 shows the clef and notation legend I have created that indicates where notation for each limb appears on the staff and Figure 13.4 provides an example of how my notation is used to define the pad number to be struck, the necessary rhythmic phrasing that must be followed to ensure that sounds are activated at the correct time, and the coordination required to perform the piece. This approach provides a flexible solution to notating performances that would work for controllers made by alternative manufacturers with different numbers of pads or kit configurations.

The multi-stave method used by Jimbo (2003) (mentioned earlier in this chapter), only works effectively when using MIDI note numbers to trigger the specific corresponding melodic pitch, i.e., a note number of 60 will play $\mathrm{C} 3$. This approach does not translate well to sample-based projects where any pitch or sound can be activated using any MIDI note number.

With the addition of a standard drum kit staff, the notation method also works effectively for hybrid setups (Figure 13.5).

This notation method is highly effective in addressing how to perform a piece. However, until there is an easy way to automatically replicate the technical setup of the equipment originally used to develop the project, on technologies or software from a different manufacturer, the sharing of these projects remains problematic; these challenges are discussed further, below. 


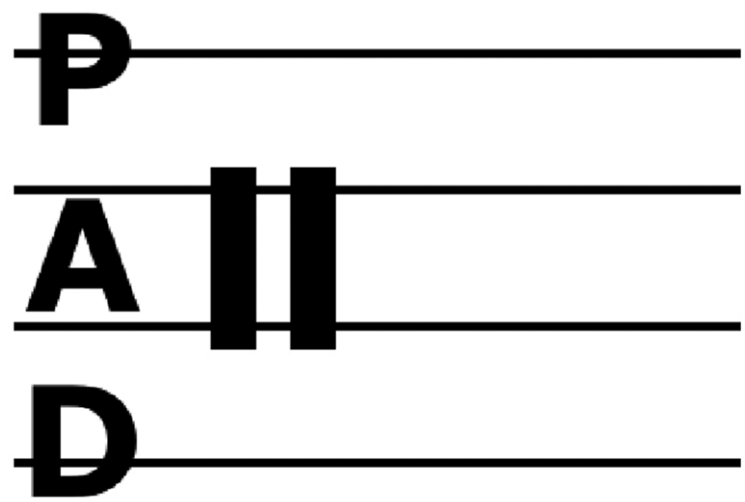

\section{Right Hand Left Hand Right Foot Left Foot}

Figure 13.3 Notation legend.

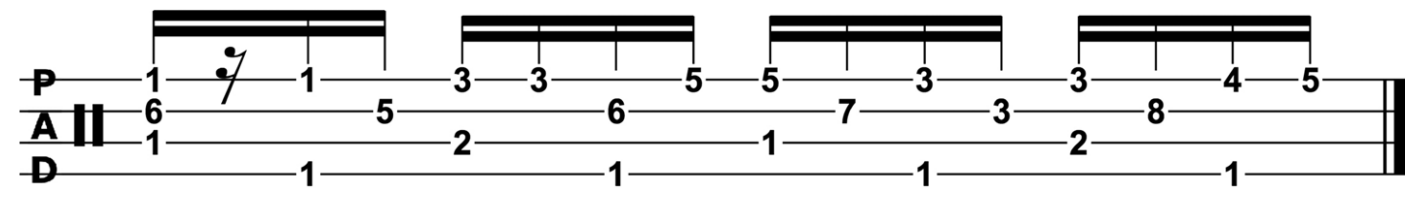

Figure 13.4 Example of percussion controller notation to suit the electronic setup in Figure 13.2.

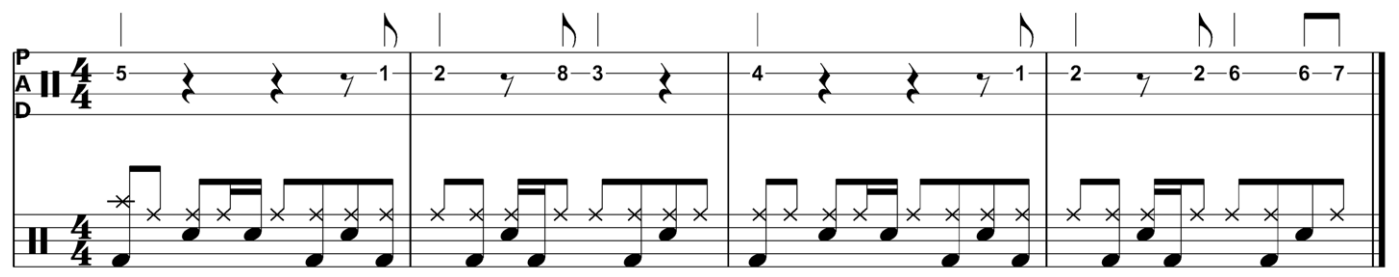

Figure 13.5 Notation for a Hybrid kit setup.

\section{Summary}

With greater numbers of drummers integrating technology into their setups it is essential that these technologies become a common feature in drum kit curricula, and that drum kit students leave university with a strong knowledge of drum kit related technologies and their potential for creative application. Whilst the curriculum and associated learning and teaching I have designed focus on the use of Roland products and Apple's Logic Pro X, the content and learning are entirely portable and relevant to the drum kit, hardware and software technologies produced by other manufacturers. The curriculum explores the language of MIDI, as for most music technologies this is the only way to activate devices produced by different manufacturers. By learning the relevant MIDI functionality, connectivity, parameters, and commands, students can apply this knowledge in any drum kit technology setting. 
The decision to move much of the "static" curricular content into the online learning environment ensures that students can revisit content to prepare better for class and be ready to deploy the learning in a practical setting. Utilizing problembased learning in face-to-face sessions encourages students to activate their learning in a supportive classroom environment, to devise solutions to issues that may impinge on the practicality of performances, and ensures students have the confidence to use drum kit technologies in their own performance work. Through development of their solo electronic performance projects, learners develop a deeper understanding of how technologies function and how they can be used to enhance and extend the creative possibilities in performance.

The development of a notation method should allow for wider sharing of these types of project. However, in order to make the sharing process more efficient, a method of converting the configuration data saved from a specific controller and remapping it for that of a different manufacturer would need to be developed. Additionally, a method for sharing projects that use sample controllers should also be explored, as the sample controller-based approach removes the reliance on having identical music software. Furthermore, deciphering the way in which a piece has been performed on pads (i.e., was pad 1 struck with the right or the left stick?) that enables it to be easily notated, is particularly challenging. This will require future research and product development with drum kit technology manufacturers in order to find a workable solution.

\section{Notes}

1 Sample pads are self-contained units with a playing surface consisting of multiple rubber pads. Each of the pads on the playing surface can activate either internal or user-created audio samples. Examples of these units are the Roland SPD-SX, Yamaha DTX Multi 12, and the Alesis Sample Pad Pro. These can be connected via MIDI (Musical Instrument Digital Interface) to third-party software or hardware to activate other sounds.

2 Percussion controllers are self-contained units that have a playing surface consisting of multiple rubber pads that when struck can activate internal factory preset sounds only. Examples of these units are the Roland SPD-20, Alesis Performance Pad, and the Nord Drum 3P. These units can be connected via MIDI to third-party software or hardware to activate other sounds.

3 Simmons drums were produced between 1980 and 1994 (and have since been reimagined for the contemporary era, with a new line appearing for the US market in 2017). These were fully electronic drum kits that resembled the typical acoustic drum kit setup. The 1980-1994 kits were recognizable by their hexagonal pads.

4 Music software packages such as Logic Pro X and Ableton Live.

5 The Roland SPD-20, first released in 1998, is a single unit containing eight playable rubber pads that can each be used to either activate internal sounds or to send MIDI information to 
an external device (such as a synth) to activate external sounds. The SPD-20 also has four trigger inputs allowing up to eight additional pads to be added to the peripheral setup.

6 Trigger pads have a playing surface generally made of rubber or mesh that when struck, output or affect an electrical signal. If connected to a drum trigger module this electrical signal can be used to activate a sound. These are not to be confused with acoustic drum triggers, which use the vibrations of an acoustic drum to activate sounds in a trigger module.

7 The instrument is sometimes taught as an ensemble instrument focusing on integration and support for a band. The outcome for students studying one-to-one with a tutor that focuses primarily on teaching advanced instrument specific skill sets will likely be very different.

8 The hybrid setups used by these drummers allow them to emulate, or reproduce, the sounds created in the studio by producers in a live environment.

9 External examiners are employed within the UK's higher education sector to ensure that assessments are fair, relevant, and meet the subject benchmark expectations at the appropriate level as set out by the national Quality Assurance Agency for Higher Education (QAA).

10 This is achieved by simply substituting a pad strike for a strike that would naturally occur in a standard acoustic drum groove or by using the bass drum pattern to simultaneously activate a bass guitar note.

11 Setups that use only trigger pads and percussion controllers with music software. These no longer conform to the standard drum kit layout and can be used to activate any recorded sound with any limb.

12 The London College of Music is a music examination board. They provide a framework of exams that are used to benchmark and assess the level of both practical musicianship and/ or music theory from beginner level to the advanced musician.

13 AVID is the parent company that designs, supplies, and supports the music software packages Pro Tools and Sibelius. AVID offers professional accreditation and certification pertaining to use of each of their software packages.

14 Students have often had prior experience of the Sibelius music scoring software at secondary school, hence why they can achieve the more advanced 110 qualification by the end of Year 1.

15 The SPD-20 contains ninety-nine patches. Each patch can contain a different configuration of MIDI settings, internal sounds and effects.

16 The constituent instruments of the arrangement have been exported as audio files and loaded into a software sampler. They have then been mapped using MIDI to pads on the SPD-20 to allow the piece to be performed by a single player using a hybrid drum kit setup that incorporates the SPD-20 percussion controller.

17 These elements of the curriculum include: software functionality, hardware functionality; MIDI and how it relates to software and hardware; and learning how to perform "Spitfire" by The Prodigy.

18 This includes how to connect the SPD-20 via MIDI to the computer, accessing internal sounds on the SPD-20, accessing and adjusting the MIDI parameters on the SPD-20 and how these signals are processed in Logic Pro X music software. 
19 The Roland SPD-20 percussion controller has been chosen due to its: MIDI functionality; four stereo connections for external trigger pads; two MIDI notes numbers on different MIDI channels can be sent from a single pad; and the speed of setup after a patch change. The MIDI output functionality allows the SPD-20 to be connected to an external device such as a computer to access and activate a wider variety of sounds. This also allows for multiple instruments to be activated when a single pad is struck. Four trigger inputs allow the units eight pad playing surface to be extended by adding as many as eight external trigger pads, thus increasing the number of sounds that can be activated on a single patch. The speed of patch changes and setup ensures that the performer can make use of every note in the bar rather than having to wait for the unit to load information before the player can continue with the performance.

20 This refers to using MIDI parameters, such as single MIDI notes, to activate functions in Logic X such as software instrument automation, Touch Tracks, and the Chord Memorizer.

21 This refers to the way instruments might be grouped together when they are exported from music software as a single consolidated sample or how a melody might be activated from a series of individual pads using MIDI.

22 MIDI Program Changes can be used to change patch on the SPD-20. A sequence of Program Changes placed on a MIDI track can be used automate the patch changes on behalf of the performer.

23 Foot switches could be used as tools to shift through patches; however, foot switches are less flexible than MIDI Program Changes and, in this setting, do not provide the timing accuracy required to realize these projects. Most controllers have a patch chain function that allows the user to arrange patches in a specific order, but there is a limit to the number of patches that can be contained in the chain (sixteen per chain on the SPD-20). In some cases, students require sixteen patch changes just to perform the verse of their chosen song, so this limit precludes the use of this function.

\section{References}

Bergmann, Jonathan and Aaron Sams (2012), Flip Your Classroom: Reach Every Student in Every Class Every Day, Eugene, OR: International Society for Technology in Education. Bruford, Bill (1982), Bruford and the Beat, Baltimore: Axis Video, videocassette (VHS). Dean, Matt (2012), The Drum: A History, Plymouth: Scarecrow Press.

Edinburgh Napier University (n.d.), Edinburgh Napier University Strategy 2020. https://view. joomag.com/strategy-2020-building-success/0536670001429606029?short (accessed November 20, 2018).

Garrison, D. Randy and Terry Anderson (2003), E-Learning In the 21st Century: A Framework for Research and Practice, London: Routledge.

Garrison, D. Randy and Heather Kanuka (2004), "Blended Learning: Uncovering its Transformative Potential in Higher Education," The Internet and Higher Education, 2004, 7 (2): 95-105. 
Hartley, Peter and Ruth Whitefield (2011), "The Case for Programme Focused Assessment," Educational Developments, 12 (4): 8-12.

Jimbo, Akira (2003), Fujiyama: Combining Acoustic and Electronic Drums, New York: Carl Fischer.

Lave, Jean and Etienne Wenger (1991), Situated Learning: Legitimate Peripheral Participation, Cambridge: Cambridge University Press.

McDowell, Liz (2012), Programme Focused Assessment: A Short Guide. Available online: www.brad.ac.uk/pass/resources/short-guide.pdf (accessed November 15, 2018).

Meskill, Carla (2013), Online Teaching and Learning: Sociocultural Perspectives, London: Bloomsbury.

Selwyn, Neil (2011), Education and Technology: Key Issues and Debates, London: Continuum.

Smith, Gareth Dylan (2013), I Drum, therefore I Am: Being and Becoming a Drummer, Farnham: Ashgate.

Smith, Gareth Dylan (2014), "Popular Music in Higher Education," in Graham Welch and Ioulia Papageorgori (eds.), Advanced Musical Performance: Investigations in Higher Education Learning, 33-48, Farnham: Ashgate.

Terry, Bob (2011), The Beginners Guide to Electronic Drums, Milwaukee, WI: Hal Leonard Corporation.

The Percussive Arts Society (1984), Percussive Arts Society International Drum Rudiments. Available online: www.pas.org/docs/default-source/default-document-library/ pasdrumrudiments2015.pdf (accessed February 19, 2018).

The Prodigy (2004), "Spitfire," track 1 on Always Outnumbered Never Outgunned, XL Recordings, compact disc.

Vygotsky, Lev S. (1978), Mind in Society: The Development of Higher Psychological Processes, Cambridge, MA: Harvard University Press. 